\title{
Nonstationary Electric Drifts in the Solar Atmosphere
}

\author{
I.S. Veselovsky \\ Institute of Nuclear Physics, Moscow State University
}

\begin{abstract}
Induced electric fields and nonstationary drift motions are considered. Nonstationary electric drifts may be important in erupting prominences, rising and shrinking coronal loops, and other phenomena in the solar atmosphere. Theoretical limitations are indicated for the concept of dominant plasma motions along magnetic field lines.
\end{abstract}

\section{Introduction}

Electric fields are difficult to measure in the solar atmosphere and are poorly known. Electric fields are generated in two ways: the solenoidal part depends on the electric current time variations and the potential or polarization part is due to electric charging. Electric charges and polarization fields are not invariant against coordinate system transformations. Because of this, the quasi-neutrality of the current carrying medium is dependent on the reference frame. The preferred reference system, where it is valid, should be always indicated to avoid confusion. The neglect of these facts and improper handling of electric fields and "frozen-in conditions" may sometimes lead to physically wrong general conclusions about slow plasma motions across magnetic fields in the solar atmosphere.

The purpose of this paper is to show that solenoidal electric fields are especially important when considering nonstationary motions in the solar atmosphere. Solenoidal electric fields are also termed nonpotential or induced electric fields.

\section{Induced Electric Fields and Drifts}

Eddies in the electric field $E(\vec{r}, t)$ are generated by time dependent magnetic fields $B(\vec{r}, t)$ according to Faraday's law

$$
\operatorname{rot} \vec{E}=-\frac{1}{c} \dot{\vec{B}}
$$

Equation (1) leads to the expression for the induced electric field

$$
\vec{E}_{\text {ind }}=-\frac{1}{4 \pi c} \int[\dot{\vec{B}}(\vec{r}, t) \times \vec{R}] R^{-3} d^{3} r^{\prime}
$$

where $\vec{R}=\vec{r}-\vec{r}$. The induced electric field (2) may be represented using the vector-potential $\vec{A}(\vec{r}, t)$ by the formula $\vec{E}_{i n d}=-\frac{1}{c} \vec{A}$ because of $\vec{B}=\vec{\nabla} \times \vec{A}$. Induced electric fields are often (but not always) dominant in space plasma 
conditions because of the strong magnetic fields which are varying with time. Potential electric fields $\vec{E}_{\text {pot }}=-\nabla \varphi$ are completely determined by the charge density distribution $\rho(\vec{r}, t)$ in space according to the equation $\varphi=\int \rho R^{-1} d^{3} r^{\prime}$. Because of this, potential electric fields are important when free charges are separated at sufficiently small spatial scales. The total electric field is equal to the sum of the two parts, the induced and potential ones: $\vec{E}=-\frac{1}{\mathrm{c}} \vec{A}-\nabla \varphi$.

We will consider the case of small electric potentials and large quasistationary varying magnetic fields in the laboratory frame. Magnetic fields in this case are completely determined by the electric currents $\vec{j}(\vec{r})$ according to the Bio-Savart law:

$$
\vec{B}=\frac{1}{c} \int\left[\vec{j}\left(\vec{r}^{\prime}\right) \times \vec{R}\right] R^{-3} d^{3} r^{\prime}
$$

which follows from the second Maxwellian equation

$$
\operatorname{rot} \vec{B}=\frac{4 \pi}{c} \vec{j}+\frac{1}{c} \dot{\vec{D}}
$$

in the nonrelativistic approximation $(v \ll c)$ when displacement currents are negligible $|\dot{\vec{D}}| \ll 4 \pi \vec{j}$ and the corresponding wave lengths are large compared with the characteristic length scales (Landau and Lifshits 1983).

Electromagnetic and mechanical quantities are related to each other by material equations. The simplest example of such a relation is represented by Ohm's law

$$
j_{i}=\sigma_{i k}\left(E_{k}-\frac{1}{c} e_{k l m} v_{l} B_{m}\right)
$$

with a scalar conductivity $\sigma, \sigma_{i k}=\sigma \delta_{i k}$, which is valid only for sufficiently frequent collisions and will be used for illustration purposes. In this case

$$
\vec{j}=\sigma\left(\vec{E}+\frac{1}{c}[\vec{v} \times \vec{B}]\right)
$$

and the drift velocity of the medium across the magnetic field is equal to

$$
\vec{v}_{\perp}=c \frac{[\vec{E} \times \vec{B}]}{B^{2}}-\frac{c}{\sigma} \frac{[\vec{j} \times \vec{B}]}{B^{2}} .
$$

Drift motions are completely determined when the electric and magnetic fields are known together with the electric currents and conductivity distributions in space.

For example: a) $\vec{j}=0, \sigma \neq 0$. In the current-free medium with a finite conductivity from (7) and (8) immediately follows

$$
\vec{v}_{\perp}=c \frac{[\vec{E} \times \vec{B}]}{B^{2}}, \quad \vec{E}=-\frac{1}{c}[\vec{v} \times \vec{B}]
$$

b) $\sigma \rightarrow \infty, \vec{j} \neq \infty$. The well known frozen-in conditions (9) are valid only in this limiting case. c) $\vec{v}=0$. Ohm's law in this case has the form $\vec{j}=\sigma \vec{E}$. 
d) It is interesting to note that $\vec{v}_{\perp}=c \frac{[\vec{E} \times \vec{B}]}{B^{2}}$ only if $|\vec{j}| \ll|\sigma \vec{E}|$. Moreover, $\vec{v}_{\perp}=0$ if $|\vec{j}|=|\sigma \vec{E}|$, and $\vec{v}_{\perp}=-\frac{c}{\sigma} \frac{[\vec{j} \times \vec{B}]}{B^{2}}$, when $|\vec{j}| \gg|\sigma \vec{E}|$. We do not consider here more complicated material equations (anizotropic conductivity, generalized Ohm's law, etc.)

The frozen-in condition is often met in the solar atmosphere. Because of this drift velocities can be estimated from Equations (9) using Equation (2) as follows

$$
v_{\perp} \simeq 10^{3} \tilde{N} r t^{-1}
$$

where $v_{\perp}(\mathrm{km} / \mathrm{s}), r(\mathrm{Mm}), t(s), \tilde{N}=\tilde{B} / B_{\text {tot }}$. Here $r$ and $t$ are the characteristic space-time scales of the magnetic field variations. The total magnetic field $B_{\text {tot }}$ is represented as a sum of the background stationary field $B_{0}$ and the time variable part $\tilde{B}(t): B_{\text {tot }}=B_{0}+\tilde{B}(t)$. For an active region on the Sun with $r \sim 10-100$ $\mathrm{Mm}, t \sim 10^{4} \mathrm{~s}, \tilde{N} \sim 1$ one obtains drift velocities $v_{\perp} \sim 1-10 \mathrm{~km} / \mathrm{s}$.

Our considerations indicate that drift velocities are generally increasing with the distance from a source of quasistationary varying magnetic perturbations. Ascending prominences, coronal transients, expanding or contracting loops in the solar atmosphere often show the regularity of the same kind: larger loops are moving faster (Tandberg-Hanssen 1974, Illing and Hundhausen 1986). Expanding loops represent quasistationary structures with the plasma drift motions governed by the strong magnetic fields which are increasing with time. Contracting loops, according to this explanation, are driven by the decaying magnetic fields.

\section{Discussion and Conclusions}

Space-time correlations in the solar atmosphere are sufficiently large and allow us to distinguish individual structures like loops, ropes, arcades, threads, jets, rays, sheets, etc. The anisotropy introduced by the magnetic fields produces elongated structures with characteristic length scales $r_{\perp} \ll r_{\| \cdot}$. For nearly incompressible (subsonic) regimes one obtains from the continuity equation $v_{\|} \sim \frac{r_{1}}{r_{\perp}} v_{\perp}$, which means the visible dominance of parallel motions. More numerous regimes of compressible motions depend on the ratio of time scales $\frac{t_{1}}{t_{\|}}$; where $t_{\perp}^{-1}=v_{\perp} / r_{\perp}, t_{\|}^{-1}=v_{\|} / r_{\|}$are the characteristic times of flight across and along the field. Different solutions were described for large and small values of the duration of the nonstationary processes under consideration (flows along the magnetic tubes, local contractions and rarefactions) (Veselovsky 1991). Nonstationary drift motions and density enhancements in a time-variable dipole magnetic field were investigated under several assumptions in the three-dimensional and two-dimensional cases (Syrovatskii 1969, Gorbachev and Kellner 1988, Filippov 1996).

The results show that the concept that motions of the plasma in the regions of the solar atmosphere with appreciable magnetic fields are taking place mainly along magnetic field lines which was introduced about half a century ago (Cowling 1953, 1957) has theoretical limitations and contradicts observations in many instances: erupting prominences, twisted ropes, rising and shrinking 
coronal loops, the solar wind flow across Archimedian spirals of the magnetic field, especially in the outer heliosphere.

We conclude that the popular idea that plasma can not move freely across magnetic fields is tenable only in the cases when there are no electric drifts.

Acknowledgments. The author is grateful to the Scientific Organizing Committee of IAU Colloquium 167 cochaired by B. Schmieder and D. Rust for partial financial support and to S. Koutchmy for his kind assistance facilitating the attendance at the meeting. Useful corrections to the text by the editors are gratefully acknowledged.

\section{References}

Cowling, T.G. 1953, in The Sun, G.P. Kuiper (ed.), The University of Chicago Press, Chicago, p. 532

Cowling, T.G. 1957, Magnetohydrodynamics, Interscience: N.Y

Gorbachev, V.S. and Kellner, S.R. 1988, Sov. Phys. JETP, 94, 89

Filippov, B.P. 1996, A\&A, 313, 277

Illing, R.M.E. and Hundhausen, A.J. 1986, J. Geophys. Res., 91, 10951

Landau, L.D. and Lifshits, E.M. 1982, Electrodynamics of Continuous Media, Nauka: Moscow (in Russian)

Syrovatskii, S.I. 1969, Astrophys. Space Sci., 4, 240

Tandberg-Hanssen, E. 1974, Solar Prominences, D. Reidel: Dordrecht, Holland Veselovsky, I.S. 1991, Solnechnye Dannye, 11, 89 (in Russian) 